\title{
BMJ Open Impact of the introduction of falls risk assessment toolkit on falls prevention and psychotropic medicines' utilisation in Walsall: an interrupted time series analysis
}

Mohammed Ibrahim Aladul (D) , ${ }^{1,2}$ Bharat Patel, ${ }^{3}$ Stephen Robert Chapman (1) ${ }^{4}$

To cite: Aladul MI, Patel B, Chapman SR. Impact of the introduction of falls risk assessment toolkit on falls prevention and psychotropic medicines' utilisation in Walsall: an interrupted time series analysis. BMJ Open 2021;11:e039649. doi:10.1136/ bmjopen-2020-039649

- Prepublication history for this paper is available online. To view these files, please visit the journal online (http://dx.doi. org/10.1136/bmjopen-2020 039649).

Received 21 April 2020 Accepted 20 July 2021

Check for updates

(c) Author(s) (or their employer(s)) 2021. Re-use permitted under CC BY-NC. No commercial re-use. See rights and permissions. Published by BMJ.

${ }^{1}$ Pharmacy College, Department of Clinical Pharmacy, University of Mosul, Mosul, Iraq

${ }^{2}$ Pharmacy College, Ninevah University, Mosul, Iraq

${ }^{3}$ Rushall Medical Centre, Walsall, UK

${ }^{4}$ School of Pharmacy, Keele University, Newcastle under Lyme, UK

Correspondence to

Dr Mohammed Ibrahim Aladul; m.i.m.aladul@uomosul.edu.iq

\section{ABSTRACT}

Objective To determine the impact of the introduction of a falls risk assessment toolkit (FRAT) in a UK medical centre on the number and cost of non-elective admissions for falls and psychotropic medication utilisation.

Design Interrupted time series analysis quantifying the number and cost of non-elective admissions for falls and primary care use data for Rushall Medical Centre before and after the implementation of FRAT at July 2017. Setting Data on the monthly number and cost of nonelective admissions for falls and number of referrals and assessment to the falls service were provided by Walsall Clinical Commissioning Group. Primary care prescribing cost and volume data for Rushall Medical Centre was derived from the Openprescribing.net website for prescriptions dispensed between April 2015 and November 2018.

Primary and secondary outcome measures The number and cost of non-elective admissions for falls and number of referrals and assessment to the falls service, and the volume of utilisation of psychotropic medicines. Results Following the implementation of FRAT at Rushall Medical Centre in July 2017, the number of non-elective admissions for falls decreased at a rate of 0.414 admissions per month $(\mathrm{p}<0.033,95 \% \mathrm{Cl}-0.796$ to -0.032). The utilisation of psychotropic medications (alimemazine, citalopram, escitalopram, fluoxetine, mirtazapine, olanzapine and risperidone) decreased. The expenditure on psychotropic medications prescribed/used at Rushall Medical Centre decreased by at least $£ 986$ per month $(p<0.001,95 \% \mathrm{Cl}-2067$ to -986$)$.

Conclusions The implementation of FRAT at Rushall Medical Centre was associated with a reduction in the number of non-elective admissions for falls. Assessment of these patients together with deprescribing of psychotropic medications resulted in a reduction in the number of nonelective admissions for falls and associated costs.

\section{INTRODUCTION}

Falls and fall-related injuries are one of the leading causes of injury and emergency hospital admission in the UK. ${ }^{1}$ The global annual risk of falling increases with ageing, from $28 \%-35 \%$ for patients aged $>64$ years to
Strengths and limitations of this study

- Segmented regression of an interrupted time series is regarded as the gold standard in pharmacoepidemiological studies.

- Openprescribing.net website provides a reliable source for prescriptions dispensed in England.

- National data (if available) would give a more comprehensive view of the impact of the tool.

$32 \%-42 \%$ for those aged $>70$ years. The WHO has warned that these figures will double by 2030 if no effective fall-prevention strategies adopted urgently. ${ }^{2}{ }^{3}$ In England, the Public Health Outcomes Framework reported that in 2017-2018 there were 220160 emergency hospital admissions related to falls among patients aged $\geq 65$ years, with around $67 \%$ of these patients aged $\geq 80$ years. ${ }^{4}$

The implications of falls or fall-related injuries on patients' quality of life, including loss of independence, pain and even mortality, ${ }^{5}$ have been estimated to cost the National Health Service (NHS) about £2.3 billion annually. ${ }^{6}$ The finite amount of healthcare resources limits the provision of interventions or referrals services for all communitydwelling older patients at risk of falling. For this reason, the National Institute for Health and Care Excellence (NICE) suggests that patients at highest risk of falling are targeted to benefit most from intervention. ${ }^{67}$ Reviews show that fall-prevention interventions can be cost saving. ${ }^{8}$

Falls risk assessment tools are designed to identify people at high risk of falls to allow for cost-effective targeting of fall-prevention strategies. ${ }^{9}$ The term 'falls risk assessment tool' describes many different types of tools which includes numerical risk prediction tools, fall risk factor checklists, multifactorial 
assessment or functional mobility assessments, that are aimed to predict the risk of falls. ${ }^{10}$

Falls risk assessment tools used in primary care generally are multifactorial and are normally paper-based. ${ }^{11}$ One of the commonly used tools, referred to in the American Geriatrics Society/British Geriatrics Society guidelines for test of balance and gait only, in primary care includes the 'Timed Up and Go' test. ${ }^{12}{ }^{13}$ Although practical and easy to use in any setting, a review by Barry et $a l^{14}$, concluded that the test has limited predictability for assessing high risk of falling and should not be used in isolation.

The use of computerised prompts in general practitioner (GP) consultation has become a routine component of a clinician and patient interaction. ${ }^{15}$ The most common UK general practice computer software, Egton Medical Information Systems (EMIS; EMIS is an electronic patient record system used across primary care, community care and mental health) allows for the design of audit templates which can be either proactive or reactive. ${ }^{16}$ There are variations of falls risk assessments available for the community-dwelling older population with differing combinations of risk factors, but few have published evidence of validity and reliability. ${ }^{6} 1117$

The falls risk assessment toolkit (FRAT) is a computerbased risk assessment tool that incorporates risk stratification criteria (searches/queries) consistent with national guidance. The FRAT was developed by the Centre for Medicines Optimisation at Keele University and the Medicines Management team at Walsall Clinical Commissioning Group (CCG) and was endorsed by $\mathrm{NICE}^{6}$; it is integrated with EMIS and identifies high-risk patients regardless of whether they have had a fall. ${ }^{18}$

The components of FRAT include a protocol, a filtering method for risk identification using pre-existing parameters (age $>65$ years, history of falls and psychotropic medication), and a template of multiple questions equivalent to a multifactorial risk assessment. The protocol and template are linked to the system. An alert appears when a patient is identified as a high risk by the protocol criteria. Figure 1 is an example of the EMIS web template which contains multiple categories for falls risks. The FRAT allows for either continuous background searching of all patients or proactive case finding which involves screening the GP practice patient database.

The FRAT was piloted in January 2016 and made nationally available in April 2016. An audit of the FRAT identified 35 patients from 30000 which estimated that it detects about 1 in 1000 high-risk patients. ${ }^{19}$

The aim of this study is to determine the impact of the introduction of FRAT on the number of non-elective admissions for falls at a UK medical centre and the effect on utilisation of psychotropic medications.

The interrupted time series analysis is considered as the most robust quasiexperimental design in drug utilisation studies. The strength of this design lies in its ability to evaluate the effects of interventions for which it is difficult to identify an appropriate control group. ${ }^{20}$ Therefore, to address the study aims, the interrupted time series analysis was used to identify the impact of the implementation of FRAT on the number and the cost

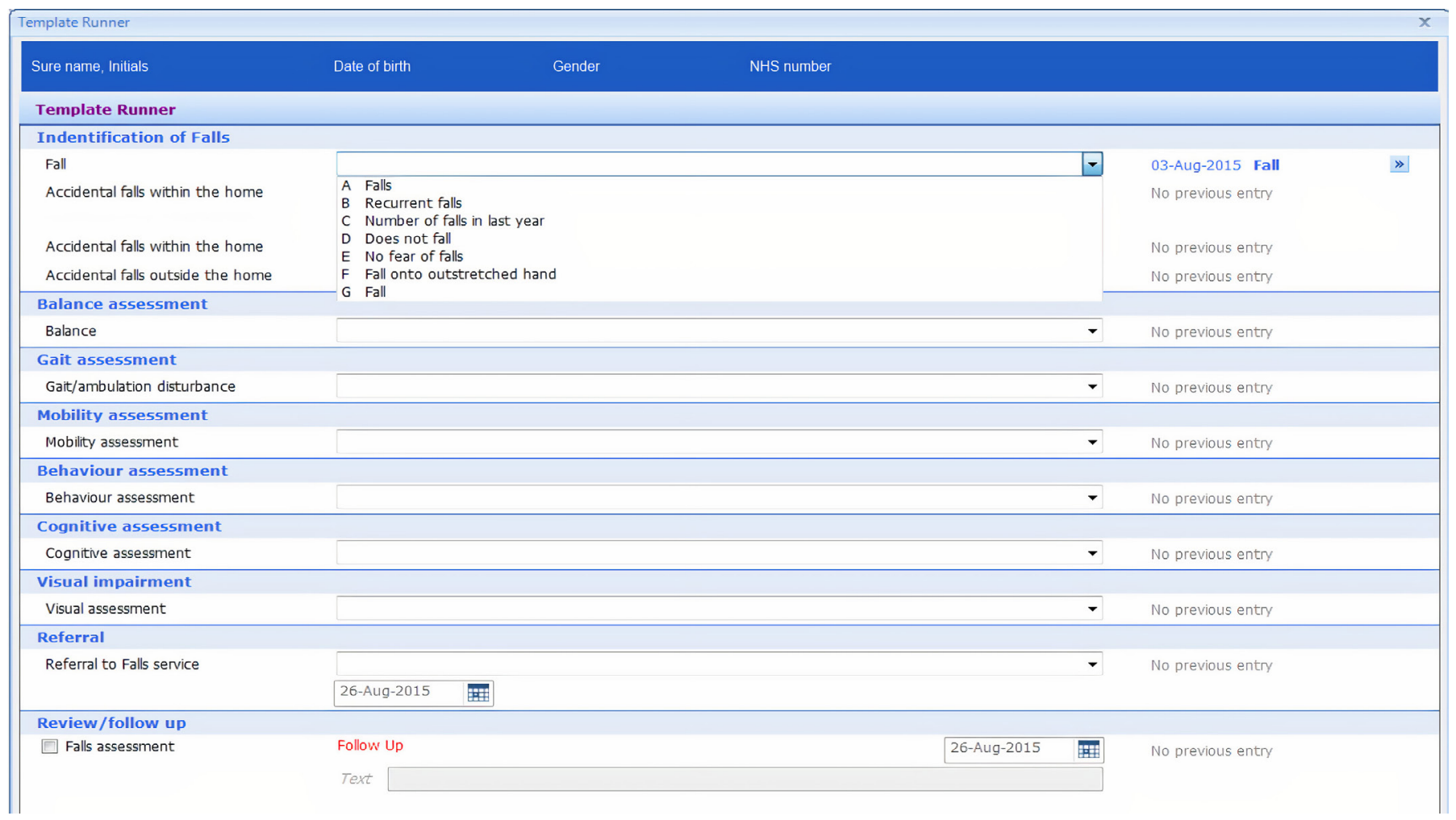

Figure 1 Screenshot of the FRAT for a virtual patient. FRAT, falls risk assessment toolkit. 
of monthly non-elective admissions for falls at Rushall Medical Centre as well as the volume of utilisation and expenditure on psychotropic medications.

\section{METHODS}

In June 2017, Rushall Medical Centre started to implement FRAT (which was integrated with EMIS web) to screen and identify older patients with a chance of falling within the next 5 years. Identified patients who were potentially suspected to fall were contacted and referred to the fall assessment team within the centre. These identified patients were also screened and deprescribed psychotropic medicines that may potentially lead to a fall. Deprescribing is a planned reviewing and identifying of patient's existing medications, implemented by healthcare professionals to reduce or withdraw unnecessary or potentially harmful medication to improve patient's outcome. ${ }^{21}$ The study was designed as a service evaluation to determine the ability of FRAT to screen and detect falls from a database, therefore, research ethics committee approval was not required.

\section{Primary and secondary outcome measures}

The primary outcome variables were the number of all non-elective admissions where the primary diagnosis was fall (ICD10 code W0\%-W1\%) to the falls service at Rushall Medical Centre in England together with the number of patients identified by FRAT as potentially likely to fall (within the next 5 years) and referred to the falls assessment team within the centre. Data on the monthly number and cost of non-elective admissions for falls and number of referrals and assessment to the falls service were provided by Walsall CCG. The secondary outcome variables were the monthly data of utilisation and expenditure on psychotropic medicines which were derived from the Openprescribing.net website for prescriptions dispensed between April 2015 and November 2018. ${ }^{22}$ Reviewed psychotropic medicines included 63 medicines that are recognised by the toolkit (FRAT).

The inclusion criteria were patients with a fall diagnosis (ICD10 code W0\%-W1\%) in any diagnostic position for patients aged $\geq 65$ years, with an evidence of fall (risk) or having psychotropic medication. Those who had medications review in last 3 months, between April 2015 and November 2018 were excluded.

\section{Data analysis}

Segmented regression analysis of interrupted time series was used for both the monthly number and cost of nonelective admissions for falls supplied by Walsall CCG and the monthly data on the utilisation and cost of psychotropic medicines extracted from Openprescibing.net used before and after the implementation of FRAT at Rushall Medical Centre, using the method of Wagner et $a l^{23}$ The effect was assessed by two parameters, level $(\beta 2)$ and trend ( $\beta 3)$. The following segmented regression analysis equation was applied to each individual study outcome measure:

$\mathrm{Y}_{\mathrm{t}}=\beta_{0}+\beta_{1} \times$ time $+\beta_{2} \times$ implementation of FRAT in July $2017+\beta_{3} \times$ time after implementation of FRAT in July $2017+\mathrm{e}_{\mathrm{t}}$

where $Y_{t}$ is the monthly outcome measure. Time was a continuous variable referring to time, in months, from the start of the observation period, ranging from 1 to 44 from the start to the end of the study period. The implementation of FRAT in July 2017 was a dichotomous variable ( 0 before July 2017; 1 since July 2017). Time after implementation of FRAT in July 2017 was a continuous variable beginning in July 2017. $\beta_{0}$ and $\beta_{1}$ represent the intercept and trend over time during the preintervention period, respectively. $\beta_{2}$ represents the change in the level at the time of implementation of FRAT in July 2017 and $\beta 3$ represents the trend change in the slope after implementation of FRAT in July 2017, both compared with those in the preintervention period. $\mathrm{e}_{\mathrm{t}}$ represents the error term. This analysis was performed using Stata 'ITSA' command. ${ }^{24}$ The data were tested for autocorrelation and seasonality before the analyses. Holt-Winters seasonal smoothing approach and Prais-Winsten ordinary leastsquares regression approach were used to adjust for the present seasonality and autocorrelation, respectively. ${ }^{25}$

Number of referrals to the falls' assessment team was examined in Rushall Medical Centre following the implementation of FRAT in July 2017 by linear regression analysis. Linear regression analysis was used with a month as the independent variable and the number of referrals to the falls' assessment team as the dependent variable. The regression coefficient values were divided by the baseline number of referrals (in July 2017) to calculate the average monthly percentage increase or decrease in falls at Rushall Medical Centre. All calculations were performed using Microsoft Excel 2013 and STATA MP13.

\section{Patient and public involvement}

No patients or members of the public were involved in this study.

\section{RESULTS}

Change in the number and the cost of non-elective admissions for falls

The interrupted time series analysis showed that from April 2015 to June 2017, there was no evidence of change in the level and trend of the number and the cost of nonelective admissions for falls per month $(\mathrm{p}<0.064,95 \%$ CI -0.007 to 0.257$)$ and ( $>0.876,95 \%$ CI -620 to 534 ), respectively over this period. Following the implementation of FRAT at Rushall Medical Centre in July 2017, the number of non-elective admissions for falls decreased at a rate of 0.414 admissions per month $(\mathrm{p}<0.033,95 \%$ CI -0.796 to -0.032 ; figure 2 ), while there was no evidence of a change in the cost of non-elective admission for falls per month ( $\mathrm{p}<0.087,95 \%$ CI -2738 to 186$)$. 


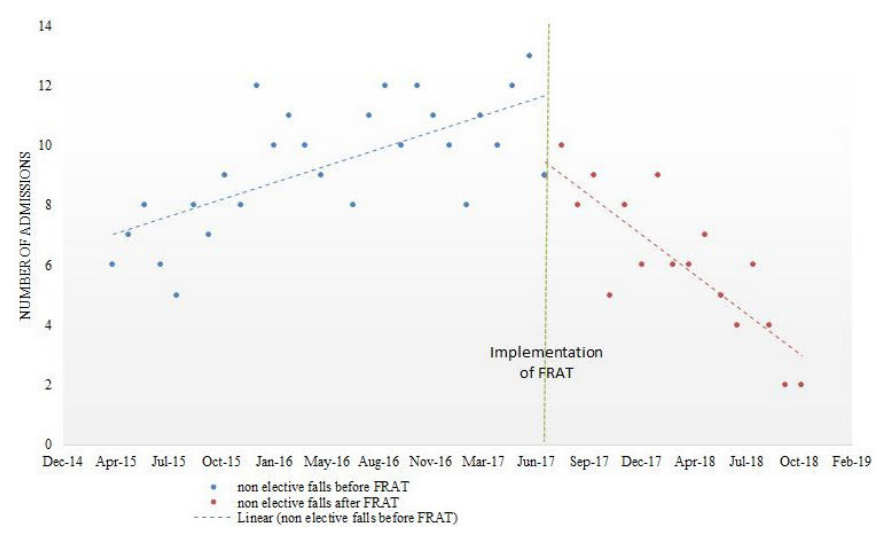

Figure 2 Change in the number of non-elective admissions before and after the implementation of FRAT. FRAT, falls risk assessment toolkit.

\section{Change in utilisation of psychotropic medications prescription/utilisation}

The interrupted time series analysis showed that from April 2015 to June 2017, there were no evidence of change in the level and trend of psychotropic medications' utilisation, namely, alimemazine, citalopram, escitalopram, fluoxetine, mirtazapine, olanzapine, benzodiazepines, chlorphenamine and risperidone, while the utilisation of quetiapine increased at an estimated rate somewhere between 2.6 and 16.7 defined daily dose per month ( $p<0.010,95 \%$ CI 2.57 to 16.7 ). Following the implementation of FRAT at Rushall Medical Centre in July 2017, the utilisation of all the aforementioned psychotropic medications decreased.

\section{Change in expenditure on psychotropic medication prescribed/used at Rushall Medical Centre}

The interrupted time series analysis showed that from April 2015 to June 2017, expenditure on psychotropic medicines prescribed at Rushall Medical Centre was $£ 35474$ per month and there was no evidence of a change in the expenditure per month $(\mathrm{p}<0.233,95 \% \mathrm{CI}-450$ to $115)$ over this period. Following the implementation of FRAT at Rushall Medical Centre in July 2017, the expenditure on psychotropic medications prescribed at Rushall Medical Centre decreased by at least $£ 986$ per month ( $\mathrm{p}<0.001,95 \%$ CI -2067 to -986$)$.

\section{Number of referrals to the falls assessment team in Rushall Medical Centre}

The linear regression analysis showed that the number of referrals of patients to the falls assessment team in Rushall Medical Centre increased by $0.41 \%$ per month $(\mathrm{p}<0.018)$ following the implementation of FRAT in July 2017.

\section{DISCUSSION}

This study showed that the implementation of FRAT at Rushall Medical Centre was associated with a reduction of the number and cost of non-elective admissions for falls and the prescribing/utilisation of psychotropic medications while the number of referrals to the falls assessment team at the surgery increased.

Before the implementation of FRAT, there were an increasing number of non-elective admissions for falls and associated costs among older patients. The cumulative cost of non-elective admissions for falls within the CCG was about $£ 600000$. This was in line with many studies' findings. ${ }^{326}$ Tian et al's ${ }^{27}$ study showed that the cost of falls (care associated with the fall itself and in the year following the fall) of only 421 older patients (aged $>65$ years) in Torbay, Wales was $>£ 5$ million, which accounted for $4 \%$ of the whole local adult social care budget in Torbay.

The implementation of this toolkit led to a decrease in the trend of non-elective admissions for falls, with subsequent reduction in the cost of non-elective admissions for falls. The utilisation of the majority of the reviewed psychotropic medicine was increasing before FRAT; there was a reduction in prescribing and increased deprescribing following FRAT implementation. Previous studies have demonstrated that a reasonable percentage of older patients (aged $>65$ years) can tolerate deprescribing of certain classes of medications without harmful consequences and with possible improvement in quality of life.$^{28}{ }^{29}$ Campbell et al $\mathrm{s}^{30}$ study found that deprescribing of psychotropic medications was associated with reduced risk of falling among older patients. Similarly, Chertkow et al's ${ }^{31}$ study also showed that benzodiazepine deprescribing would improve cognition and reduce the risk of falls. Tinetti et al's ${ }^{32}$ study suggested that deprescribing an antihypertensive medication may reduce the risk of falls in older patients. The result of this study is in line with the NICE pathway for preventing falls in older people that recommended reviewing and if possible, discontinuing (deprescribing) psychotropic medicines used by those patients, to reduce their risk of falling. ${ }^{33}$

The strength of this study is that it is the first to determine the impact of the introduction of FRAT that was endorsed by NICE and the consequent cost savings associated with falls prevention using interrupted time series analysis. The study has some limitations; it was only conducted in one medical centre and more data from other medical centres or national data would give a more comprehensive view of the impact of the tool. As with all tools, the motivation of practitioners to use it is a factor which needs to be considered and this may vary between practices. Interrupted time series analysis control for baseline trends but the internal validity of the test is reduced if other programmes began at the same time. To the best of the authors' knowledge, no competing programmes were implemented during the study period at this CCG or any change in NICE guidelines, that may confound the results. Healthcare professionals at the CCG confirmed that as far as they were aware no other similar or competing programmes were being used within the CCG. 


\section{CONCLUSION}

The implementation of FRAT at a UK medical centre was associated with automatic identification of older patients who are at a potential risk of fall and a reduction in the number of non-elective admissions for falls. Assessment of these patients together with deprescribing of psychotropic medication resulted in a reduction of non-elective admissions for falls and associated costs.

Acknowledgements The authors thank Dr Satvinder Sandilands, a senior partner at Rushall Medical Centre for her support and help in implementing the tool within the centre and access the data. MIA thanks the University of Mosul.

Contributors All authors have contributed to this study and all authors reviewed and approved the final version of the manuscript. MIA participated in the study design, data collection, interpretation of results, prepared the manuscript draft and performed all analytical testing and manuscript review. BP participated in the study design, interpreted the results and reviewed the manuscript and corrected the final version of the manuscript. SRC designed the study, interpreted the results and reviewed the manuscript and corrected the final version of the manuscript. MIA is the guarantor.

Funding The authors have not declared a specific grant for this research from any funding agency in the public, commercial or not-for-profit sectors.

Competing interests None declared.

Patient and public involvement Patients and/or the public were not involved in the design, or conduct, or reporting, or dissemination plans of this research.

Patient consent for publication Not required.

Provenance and peer review Not commissioned; externally peer reviewed.

Data availability statement Data sharing not applicable as no datasets generated and/or analysed for this study. Data are available upon reasonable request. The raw data from which result paper are derived can be made available on request.

Open access This is an open access article distributed in accordance with the Creative Commons Attribution Non Commercial (CC BY-NC 4.0) license, which permits others to distribute, remix, adapt, build upon this work non-commercially, and license their derivative works on different terms, provided the original work is properly cited, appropriate credit is given, any changes made indicated, and the use is non-commercial. See: http://creativecommons.org/licenses/by-nc/4.0/.

\section{ORCID iDs}

Mohammed Ibrahim Aladul http://orcid.org/0000-0002-7368-3469

Stephen Robert Chapman http://orcid.org/0000-0002-0326-7742

\section{REFERENCES}

1 Lincolnshire County Council (2017) emergency hospital admissions for falls (age 65 and over). Available: https://data.gov.uk/dataset/ 63f7c18d-dc38-4d30-a91c-946fd0e0ba1f/emergency-hospitaladmissions-for-falls-age-65-and-over [Accessed 5 Jul 2019].

2 Yoshida S. A global report on falls prevention, epidemiology of falls. ageing and life course, family and community health, WHO, 2007 Available: https://www.who.int/ageing/projects/1.Epidemiology\% 20of\%20falls\%20in\%20older\%20age.pdf [Accessed 5 Jul 2019].

3 Kannus P, Palvanen M, Niemi S, et al. Alarming rise in the number and incidence of fall-induced cervical spine injuries among older adults. J Gerontol A Biol Sci Med Sci 2007;62:180-3.

4 Public health England (2019) public health profiles, 2019. Available: https://fingertips.phe.org.uk/search/fall\#page/11/gid/1/pat/15/par/ E92000001/ati/6/are/E12000004 [Accessed 5 Jul 2019].

5 Ozcan A, Donat H, Gelecek N, et al. The relationship between risk factors for falling and the quality of life in older adults. BMC Public Health 2005;5:90.

6 NICE. National Institute for health and social care (2013) falls in older people: assessing risk and prevention. Available: https://www.nice. org.uk/guidance/cg161/resources/falls-in-older-people-assessingrisk-and-prevention-pdf-35109686728645 [Accessed 5 Jul 2019]

7 Todd C, Skelton D. What are the main risk factors for falls among older people and what are the most effective interventions to prevent these falls? who regional office for Europe. health evidence network report, 2004. Available: http://www.euro.who.int/document/E82552. pdf [Accessed 5 Jul 2019].

8 Gillespie LD, Robertson MC, Gillespie WJ, et al. Interventions for preventing falls in older people living in the community. Cochrane Database Syst Rev 2012;9:CD007146.

9 Healey F, Monro A, Cockram A, et al. Using targeted risk factor reduction to prevent falls in older in-patients: a randomised controlled trial. Age Ageing 2004;33:390-5.

10 Oliver D, Healey F, Haines TP. Preventing falls and fall-related injuries in hospitals. Clin Geriatr Med 2010;26:645-92.

11 Narayanan V, Dickinson A, Victor C, et al. Falls screening and assessment tools used in acute mental health settings: a review of policies in England and Wales. Physiotherapy 2016;102:178-83.

12 Podsiadlo D, Richardson S. The timed "Up \& Go": a test of basic functional mobility for frail elderly persons. J Am Geriatr Soc 1991;39:142-8.

13 Avin KG, Hanke TA, Kirk-Sanchez N, et al. Management of falls in community-dwelling older adults: clinical guidance statement from the Academy of geriatric physical therapy of the American physical therapy association. Phys Ther 2015;95:815-34.

14 Barry E, Galvin R, Keogh C, et al. Is the timed up and go test a useful predictor of risk of falls in community dwelling older adults: a systematic review and meta-analysis. BMC Geriatr 2014;14:14.

15 Kumarapeli P, de Lusignan S. Using the computer in the clinical consultation; setting the stage, reviewing, recording, and taking actions: multi-channel video study. J Am Med Inform Assoc 2013;20:e67-75.

16 EMIS. EMIS health (2019) EMIS web for primary care. Available: https://www.emishealth.com/products/emis-web/ [Accessed 5 Jul 2019].

17 Nunan S, Brown Wilson C, Henwood T, et al. Fall risk assessment tools for use among older adults in long-term care settings: a systematic review of the literature. Australas J Ageing 2018;37:23-33.

18 Keele university. Falls risk assessment toolkit, 2019. Available: https://www.fallsrisk.co.uk/ [Accessed 5 Jul 2019].

19 Chapman SR, Patel B. Identifying patients at high risk of having a fall. Clinical Pharmacist 2016;8. doi:10.1211/PJ.2016.20201031

20 Wettermark B, Martino MD, Elseviers M. Study designs in drug utilization research. In: Wettermark B, Almarsdóttir A, eds. Drug utilization research: methods and applications. 1st edn. Elseviers, 2016: 13-28.

21 Reeve E, Shakib S, Hendrix I, et al. Review of deprescribing processes and development of an evidence-based, patient-centred deprescribing process. Br J Clin Pharmacol 2014;78:738-47.

22 Open prescribing. Rushall medical centre, 2019. Available: https:// openprescribing.net/practice/M91019/ [Accessed 5 Jul 2019].

23 Wagner AK, Soumerai SB, Zhang F, et al. Segmented regression analysis of interrupted time series studies in medication use research. J Clin Pharm Ther 2002;27:299-309.

24 Linden A. Conducting interrupted time-series analysis for single- and Multiple-group comparisons. Stata J 2015;15:480-500.

25 Linden A. Using forecast modelling to evaluate treatment effects in single-group interrupted time series analysis. J Eval Clin Pract 2018;24:695-700.

26 Scuffham P, Chaplin S, Legood R. Incidence and costs of unintentional falls in older people in the United Kingdom. J Epidemiol Community Health 2003;57:740-4.

27 Tian Y, Thompson J, Buck D. Exploring the system-wide costs of falls in older people in Torbay. King's Fund, 2013.

28 Iyer S, Naganathan V, McLachlan AJ, et al. Medication withdrawal trials in people aged 65 years and older: a systematic review. Drugs Aging 2008;25:1021-31.

29 Frank C, Weir E. Deprescribing for older patients. CMAJ 2014;186:1369-76.

30 Campbell AJ, Robertson MC, Gardner MM, et al. Psychotropic medication withdrawal and a home-based exercise program to prevent falls: a randomized, controlled trial. J Am Geriatr Soc 1999;47:850-3.

31 Chertkow H, Massoud F, Nasreddine Z, et al. Diagnosis and treatment of dementia: 3. mild cognitive impairment and cognitive impairment without dementia. CMAJ 2008;178:1273-85.

32 Tinetti ME, McAvay GJ, Fried TR, et al. Health outcome priorities among competing cardiovascular, fall injury, and medication-related symptom outcomes. J Am Geriatr Soc 2008;56:1409-16.

33 NICE. Falls in older people overview, 2014. Available: http:// pathways.nice.org.uk/pathways/falls-in-older-people [Accessed 26 Jan 2020]. 